\title{
Cognitive compensatory processes of older, clinically fit patients with hematologic malignancies undergoing chemotherapy: A longitudinal cohort study
}

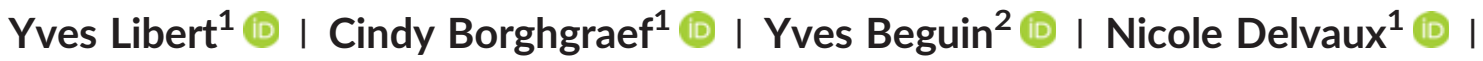

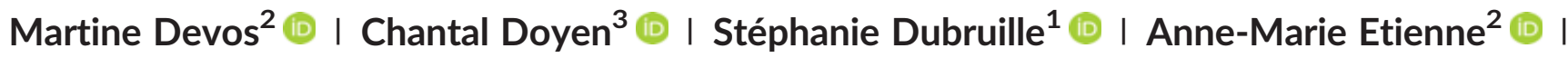 \\ Aurore Liénard $^{1}$ (i) । Isabelle Merckaert ${ }^{1}$ (i) । Christine Reynaert ${ }^{3}$ (i) । \\ Jean-Louis Slachmuylder ${ }^{1}$ (I) | Nicole Straetmans ${ }^{4}$ () । Eric Van Den Neste ${ }^{4}$ (I) । \\ Dominique Bron ${ }^{1}$ (ㄱ) । Darius Razavi ${ }^{1}$
}

${ }^{1}$ Université Libre de Bruxelles, Brussels, Belgium

${ }^{2}$ Université de Liège, Liège, Belgium

${ }^{3}$ Université Catholique de Louvain, Yvoir, Belgium

${ }^{4}$ Université Catholique de Louvain, Brussels, Belgium

Correspondence

Yves Libert, Université Libre de Bruxelles, Av. F. Roosevelt, 50-CP 191, B-1050 Brussels, Belgium.

Email: yves.libert@bordet.be

Funding information

F.R.S.-FNRS, Grant/Award Number:

7.4548 .10

\begin{abstract}
Objective: Despite the well-known negative impacts of cancer and anticancer therapies on cognitive performance, little is known about the cognitive compensatory processes of older patients with cancer. This study was designed to investigate the cognitive compensatory processes of older, clinically fit patients with hematologic malignancies undergoing chemotherapy.

Methods: We assessed 89 consecutive patients (age $\geq 65$ y) without severe cognitive impairment and 89 age-, sex-, and education level-matched healthy controls. Cognitive compensatory processes were investigated by (1) comparing cognitive performance of patients and healthy controls in novel (first exposure to cognitive tasks) and non-novel (second exposure to the same cognitive tasks) contexts, and (2) assessing psychological factors that may facilitate or inhibit cognitive performance, such as motivation, psychological distress, and perceived cognitive performance. We assessed cognitive performance with the Trail-Making, Digit Span and FCSR-IR tests, psychological distress with the Hospital Anxiety and Depression Scale, and perceived cognitive performance with the FACT-Cog questionnaire.

Results: In novel and non-novel contexts, average cognitive performances of healthy controls were higher than those of patients and were associated with motivation. Cognitive performance of patients was not associated with investigated psychological factors in the novel context but was associated with motivation and psychological distress in the non-novel context.
\end{abstract}

Conclusions: Older, clinically fit patients with hematologic malignancies undergoing chemotherapy demonstrated lower cognitive compensatory processes compared to healthy controls. Reducing distress and increasing motivation may improve cognitive compensatory processes of patients in non-novel contexts.

KEYWORDS

cancer, chemotherapy, cognitive compensatory process, older, oncology

\section{1 | INTRODUCTION}

Aging, ${ }^{1}$ cancer, ${ }^{2}$ and related treatments ${ }^{3}$ may have negative impacts on cognitive performance through multiple processes, including

Yves Libert and Cindy Borghgraef contributed equally to this work and should be considered cofirst authors. inflammation, DNA damage, or telomere shortening. ${ }^{4,5}$ Studies examining cognitive impairment in older cancer patients found that the major altered domains were processing speed, ${ }^{6-8}$ executive function, ${ }^{7,9,10}$ attention, ${ }^{9,11}$ and memory. ${ }^{7,9}$ Meanwhile, other studies reported that some older cancer patients do not develop these impairments. ${ }^{12-14}$ If these results may reflect a low sensitivity of cognitive 
task used (Mini-Mental State Examination (MMSE) or Telephone Interview of Cognitive Status), this finding may also support the hypothesis that some patients use cognitive compensatory processes, probably by recruiting additional brain areas, ${ }^{15}$ to develop and use additional resources and strategies to perform a task. Clinically, these processes could be reflected, for example, in a feeling of not being able to function as before, a need to increase mental effort for a well-known task, and difficulties to benefit from previous experience.

Although currently impossible to study directly, cognitive compensatory processes can be assessed indirectly through neuroimaging or longitudinal studies comparing cognitive performance of patients and healthy controls. In longitudinal studies, these processes should be studied by assessing the cognitive performance of older cancer patients in novel and non-novel contexts and by assessing psychological factors that may facilitate or inhibit cognitive performance. Cognitive performance in novel versus non-novel contexts may require different levels of compensatory processes. In a novel context, optimal performance requires memory skills to memorize instructions, as well as executive skills (eg, planning, inhibition, and elaboration strategies) to achieve a goal and reduce interference caused by the novel context itself. In a non-novel context, optimal performance requires the ability to remember and use previously acquired information through specific strategies, such as category clustering for memory tasks, ${ }^{16}$ leading to faster performance, in a less varied, easier manner. ${ }^{17}$ Moreover, certain psychological factors may facilitate or inhibit cognitive performance. ${ }^{18}$ This modulation reflects the use or nonuse of resources and strategies, known as cognitive compensatory processes, to optimize cognitive functioning.

There are several arguments for collecting novel and non-novel task performances to better understand cognitive compensatory processes. ${ }^{19}$ It should be recalled that, compared to a non-novel task, a novel task requires more cognitive efforts. This is certainly the case when a subject is exposed for the first time to a neuropsychological task. When a subject is re-exposed to this task, he may benefit from his previous experience. This benefit is called a "test-retest" effect. Assessing to what extent a person may benefit of such effect may inform about cognitive compensatory processes.

The aim of this study was to investigate the existence of altered cognitive compensatory processes among older, clinically fit patients with hematologic malignancies undergoing chemotherapy through a longitudinal cohort design. Patients and healthy controls, matched for age, sex, and educational level, had their cognitive performance tested twice, in novel and non-novel contexts. The first exposure of a subject to a cognitive task is considered a good proxy for exposure to a novel context. A second exposure, 1 month later, of the same subject to the same cognitive task is considered a good proxy for exposure to a nonnovel context. Influences of psychological factors, including motivation, psychological distress, and perceived cognitive performance, as potential inhibitors or facilitators of cognitive performance were assessed for both contexts. In this way, cognitive performance and its association with the chosen psychological factors were assessed longitudinally.

Beside the a priori assumption that cancer and related treatments may impair the cognitive performance of older patients, 2 hypotheses regarding the cognitive functioning of older, clinically fit patients were made to investigate cognitive compensatory processes. First, we hypothesized that, contrary to healthy controls, patients would not demonstrate improvement in cognitive performance over time (a practice effect), due to their reduced ability to use cognitive compensatory processes. Second, considering a potential floor effect related to age, cancer, and related treatments, we hypothesized that no association between patients' cognitive performance, motivation to undertake a cognitive task, psychological distress, and perceived cognitive performance would be found in a novel context, reflecting the important cognitive load required to perform a novel task and related difficulties to benefit from psychological facilitator factors. We hypothesized that, in a non-novel context, an association would be found between patients' cognitive performance and these psychological factors, reflecting the reduced cognitive load required to perform a non-novel task.

\section{2 | METHODS}

\section{1 | Patients and setting}

This study was conducted in 6 Belgian hospitals and was approved by the local ethics committees. All consecutive patient volunteers fulfilling the inclusion criteria were invited to participate and provided written informed consent. Recruited patients were at least 65 years old, had hematologic malignancies, had been admitted to begin chemotherapy, were able to speak French, and were assessed as clinically fit by their physician before treatment. The term "clinically fit" refers to the fact that the patient has no geriatric syndrome and/or irreversible comorbidity impairing daily functioning. Patients hospitalized for palliative care, those with severe cognitive impairments (as assessed by their physicians), and those who could not complete scheduled assessments for physical or psychological reasons were excluded.

Healthy controls, matched for age $( \pm 5 y)$, sex, and education level, were also recruited. To ensure that these individuals were representative of the global population of this age group, healthy controls with previous medical or surgical history (including cancer) or psychiatric disorders were not excluded. However, healthy controls with current acute medical, neurological, or psychiatric conditions were excluded. Finally, healthy controls who had previously benefitted from chemotherapy were excluded, to allow comparison between the included groups on the studied variables.

The first assessment (novel context) was conducted from the first 48 hours before the start of chemotherapy to the 48 hours after the start of chemotherapy (baseline for healthy controls). The second assessment (non-novel context) was conducted 1 month later ( \pm 15 days). Each assessment lasted approximately 1 to 1.5 hours. Except sociodemographic data, all assessment tools were given at the 2 time points.

\section{2 | Assessment tools}

\subsection{1 | Sociodemographic and medical characteristics}

Patients and healthy controls provided demographic information, including age, sex, and educational level. Physicians provided patients' medical characteristics, including diagnosis, time since diagnosis, disease status, prognosis, number of comorbidities, and hematological treatment characteristics. 


\subsection{2 | Physical functioning status}

Physical functioning status was assessed by using the following validated tools: the Karnofsky Performance Score (total score ranged from 0 to 100 ) and the Katz $\mathrm{ADL}^{20}$ (Activities of Daily Living; total score ranged from 6 to 24) and Lawton IADL ${ }^{21}$ (Instrumental Activities of Daily Living; total score ranged from 8 to 32); measures of activities of daily living (higher score indicated better physical functioning status).

\subsection{3 | Cognitive performance}

Cognitive performance was assessed through an objective assessment consisting of 3 tools that included domains likely affected by cancer and anticancer therapy. These tools were the Wechsler Digit Span Test, the Free and Cued Selective Reminding Test with Immediate Recall (FCSR-IR), and Trail-Making Test parts A and B (TMT A and B).

The Wechsler Digit Span Test (WAIS-IV) ${ }^{22}$ and a shortened version of the FCSR-IR were used to assess working and short-term memory. During the first test, digits were verbally presented to participants at a rate of 1 per second. Participants were asked to repeat digits in the same order for the forward digit span or reverse order for the backward digit span. In the current study, the number of correct trials was used. During the second test consisting of a controlled learning procedure developed by Grober and Buschke, ${ }^{23}$ participants were presented with a list of 16 written words. They were asked to associate each word with a given category cue to memorize these words (immediate recall). Thereafter, participants were invited to observe a 20 -second distractor period (count back) to prevent rehearsal. Subsequently, free recall and cued recall were assessed. This procedure was repeated after a 20-second distractor period. An alternate form was used at the second assessment. Because first and second free recalls were highly correlated, only the immediate and the second free recalls were used in this study.

The TMT $A$ and $B^{24}$ were used to assess processing speed and executive function. TMT A requires to connect 25 encircled numbers as quickly as possible. TMT B requires to alternate consecutive sequences of numbered and lettered circles. Shorter times to completion (in seconds) indicated better performance. The number of errors was also recorded. Regarding part B, as we decided to consider globally cognitive performance, it was decided to take into account both perseverative and normal errors. Because TMT A and B were highly correlated, only results of TMT B were used in this study. On the basis of these 3 tools, we calculated a global cognitive composite $z$-score consisting of a mean of 5 z-scores.

$$
\begin{aligned}
\text { CPS }_{i, j}= & \frac{z_{i, j}^{\text {Total Digit Span }}}{3}+\frac{z_{i, j}^{\text {Immediate Recall }}+z_{i, j}^{\text {Free Recall 2 }}}{6} \\
& +\frac{z_{i, j}^{T \text { MTB Time }}+z_{i, j}^{T M T B} \text { Error }(s)}{6} .
\end{aligned}
$$

$C P S_{i, j}$ is the cognitive performance score for patient $i$ in context $j$; and $z^{x}{ }_{i, j}$ is the $z$-score for variable $x$ and patient $i$ in context $j$. As higher $z_{i, j}^{\text {TMTB Time }}$ and $z_{i, j}^{\text {TMTB Error(s) }}$ indicate worse performance, these $z$ scores were reversed to calculate the global composite cognitive score.

\subsection{4 | Psychological characteristics}

\section{Motivation}

After completing perceived cognitive performance and before completing objective neuropsychological tasks, patients and healthy controls were asked to report their level of motivation to undertake these tasks on a 100-mm visual analogue scale, ranging from "not at all" to "a lot." This sequence has been chosen to fit with the objectives of this study.

\section{Perceived cognitive performance}

The FACT-Cog (Functional Assessment of Cancer Therapy-Cognitive Function) Scale is a self-reported measure of cognitive impairment and its impact on the quality of life (QOL) of patients. This 37-item translated and validated with French speaking patients with cancer ${ }^{25}$ consists of 4 subscales: perceived cognitive impairment, comments from others, perceived cognitive ability, and impact on QOL. ${ }^{26}$ Only 2 subscales, Perceived Cognitive Impairment (PCl; 18 items; score range: 0-72) and Perceived Cognitive Ability (PCA; 7 items; score range 0-28), were used in this study to calculate a global composite perceived cognitive performance z-score (PCPS), which consists of a mean of these 2 variables. A higher PCPS indicates higher perceived cognitive ability.

$$
P C P S_{i, j}=\frac{z_{i, j}^{P C I}+z_{i, j}^{P C A}}{2}
$$

\section{Psychological distress}

The Hospital Anxiety and Depression Scale ${ }^{27}$ was used to assess the psychological distress of patients and healthy controls (total score ranged from 0 to 42, higher score indicated higher psychological distress).

\section{3 | Statistical analysis}

Initially, a descriptive analysis of the 2 groups was prepared. Intergroup and intragroup comparisons of cognitive performance and psychological characteristics were computed by using nonparametric tests, as appropriate (Mann-Whitney or Wilcoxon tests). Univariate relationships between groups' cognitive performance and psychological factors were evaluated by using nonparametric tests (Spearman correlations). Group-by-time effects were analyzed by using a two-way repeated measures multivariate analysis of variance. Finally, backward stepwise linear regression models were computed to assess relationships between psychological factors and cognitive performance for each group in novel and non-novel contexts. All psychological variables with univariate $P$ values $\leq 0.05$ were entered into the model. All tests were two-tailed, and alpha was set at 0.05. Analyses were performed with SPSS software (version 23.0 for Windows; SPSS, Chicago, Illinois).

\section{3 | RESULTS}

\section{1 | Subjects}

Among 293 eligible inpatients, 98 refused to participate in the study. Twenty patients withdrew during the first assessment. In addition, 51 patients did not complete the neuropsychological assessment in the novel context and were excluded from the study. Three patients died 
TABLE 1 Participants' sociodemographic and medical characteristics

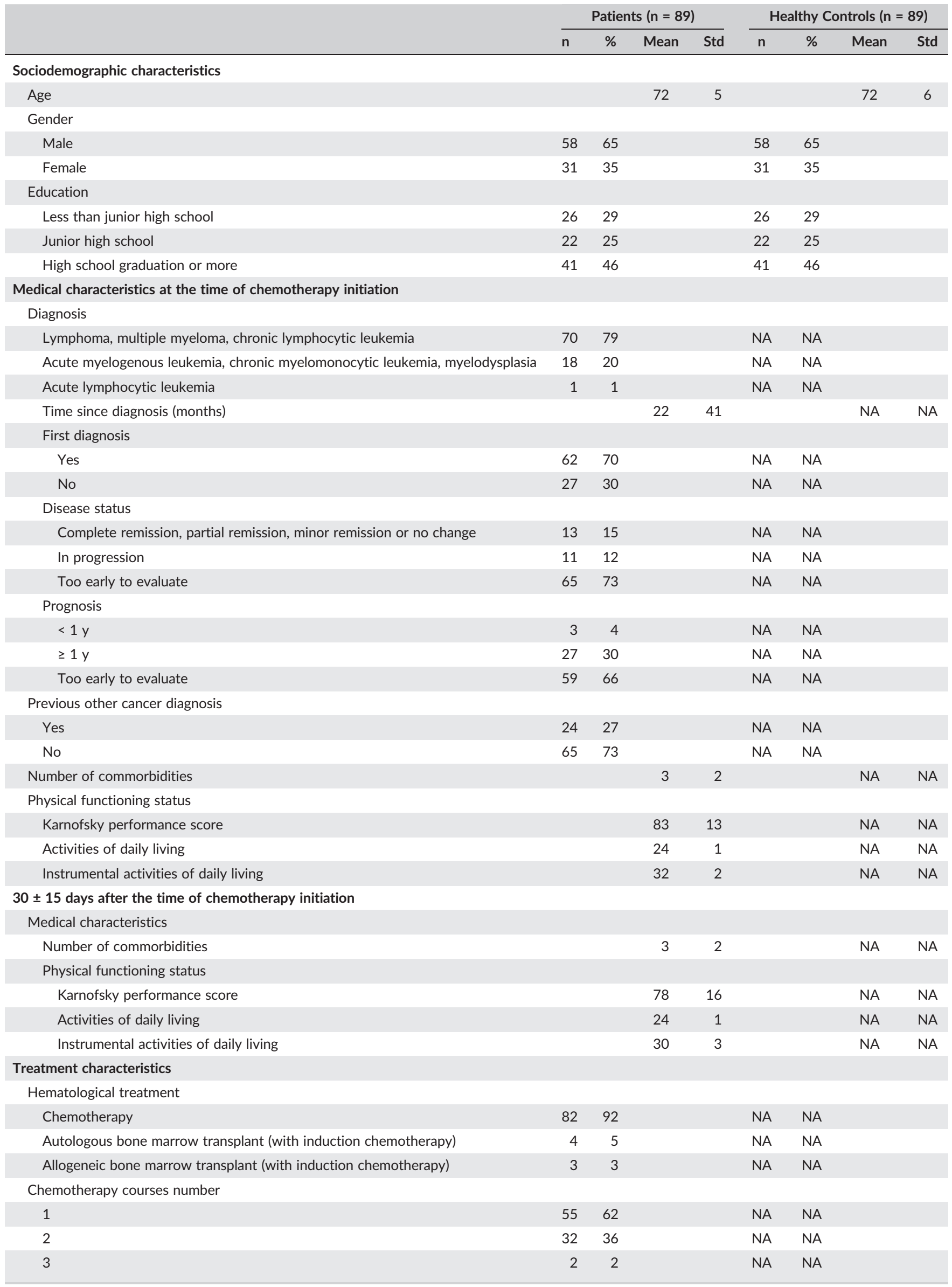


TABLE 1 (Continued)

\begin{tabular}{|c|c|c|c|c|c|c|c|c|}
\hline & \multicolumn{4}{|c|}{ Patients $(n=89)$} & \multicolumn{4}{|c|}{ Healthy Controls $(n=89)$} \\
\hline & $\mathrm{n}$ & $\%$ & Mean & $\overline{\text { Std }}$ & $\mathrm{n}$ & $\%$ & Mean & Std \\
\hline \multicolumn{9}{|c|}{ Hospitalization needed for chemotherapy } \\
\hline No & 43 & 48 & & & NA & NA & & \\
\hline Yes & 46 & 52 & & & NA & NA & & \\
\hline
\end{tabular}

Abbreviation: Std, standard deviation; NA, not applicable. No significant difference was found between patients and healthy controls regarding age $(P=0.842)$, gender (Chi-squared(1) $=0 ; P=1.000)$, and education (Chi-squared(2) $=0 ; P=1.000)$.

before the second evaluation. During the second assessment, 26 patients withdrew and 5 did not finish the complete neuropsychological assessment. One patient was excluded from statistical analysis because the interval between the 2 assessments was too long (63 days). Thus, analyses included 89 patients who were being treated in outpatient or inpatient settings, according to their diagnoses and treatments. It should be noted that patients who dropped out after the completion of the first assessment were not significantly different from included patients considering neuropsychological tasks.

Table 1 lists the sociodemographic and medical characteristics of patients. Age, sex, and educational level distributions of matched healthy controls were the same as those of patients. Table 2 displays the cognitive and psychological characteristics of participants in novel and non-novel contexts. For cognitive performance, a group effect was found, but no time or group-by-time effect was found. Group effects were found regarding psychological characteristics (perceived cognitive performance, motivation, and psychological distress). A time effect was found for perceived cognitive performance. Finally, a group-by-time effect was found for perceived cognitive performance. Table 3 lists the psychological characteristics associated with the cognitive performance of patients and healthy controls. Cognitive performance of healthy controls was associated with motivation in novel and non-novel contexts. Cognitive performance of patients was not significantly associated with investigated psychological characteristics in the novel context but was associated with perceived cognitive performance, motivation, and psychological distress in the non-novel context.

Three backward, stepwise, linear regression analyses were conducted (Table 4). For analyses of the cognitive performance of healthy controls in the novel and non-novel contexts, only motivation was entered into the models. Motivation predicted the cognitive performance of healthy controls in the novel context and non-novel context, with proportions of explained variance of $18 \%$. For analysis of the cognitive performance of patients in the non-novel context, 3 variables were entered into the model: perceived cognitive performance, motivation, and psychological distress. Performance of patients in the non-novel context was predicted by motivation and psychological distress, with a proportion of explained variance of $19 \%$.

\section{4 | DISCUSSION}

To our knowledge, this longitudinal cohort study is the first designed to investigate cognitive compensatory processes of older, clinically fit patients with hematologic malignancies undergoing chemotherapy, through the cognitive performance and associated psychological factors in novel and non-novel contexts. As expected in our hypothesis in the light of available studies about hematologic malignancies, ${ }^{28}$ healthy controls performed more optimally than patients in both contexts. The findings did not confirm our first hypothesis that patients would not improve their cognitive performance over time compared to healthy controls. Indeed, no practice effect was found for healthy controls or for patients. Our findings did confirm our second hypothesis that there would be an association between patients' cognitive performance and psychological factors in the non-novel context. A significant positive association was found between the cognitive performance of healthy controls and motivation in both contexts, whereas no significant association was found for patients in the novel context. In the non-novel context, however, a significant positive association between cognitive performance and motivation and a significant negative association between cognitive performance and psychological distress were found.

Why the results found in patients are not similar to those found in healthy controls? At a cognitive level, our results reinforce the idea that older, clinically fit patients with hematologic malignancies undergoing chemotherapy not only perform less optimally in both novel and non-novel contexts but also (1) do not improve in performance over time (healthy controls did not improve, either) and (2) do not benefit from motivation in a novel context, although low motivation and high psychological distress may decrease performance in a non-novel context. Taken together, these findings suggest that such patients have difficulty in using and/or developing additional resources to compensate for potentially altered cognitive performance due to cancer, anticancer therapy, and their psychological consequences. In other words, for these patients, it is easier to compensate for altered cognitive performance in a non-novel context, in the presence of a high motivation and a low psychological distress level, to complete cognitive tasks. It should be underlined at this level that patients have a significant higher level of psychological distress compared to healthy controls at the 2 assessment points although there is no difference in the evolution over time of psychological distress between the 2 assessments both for patients and for healthy controls.

Previous studies have emphasized cognitive changes related to cancer and chemotherapy among older patients with cancer. To our knowledge, however, this is the first study showing differences between older, clinically fit patients with hematologic malignancies undergoing chemotherapy and matched healthy controls in terms of cognitive compensatory processes in both novel and non-novel contexts. 

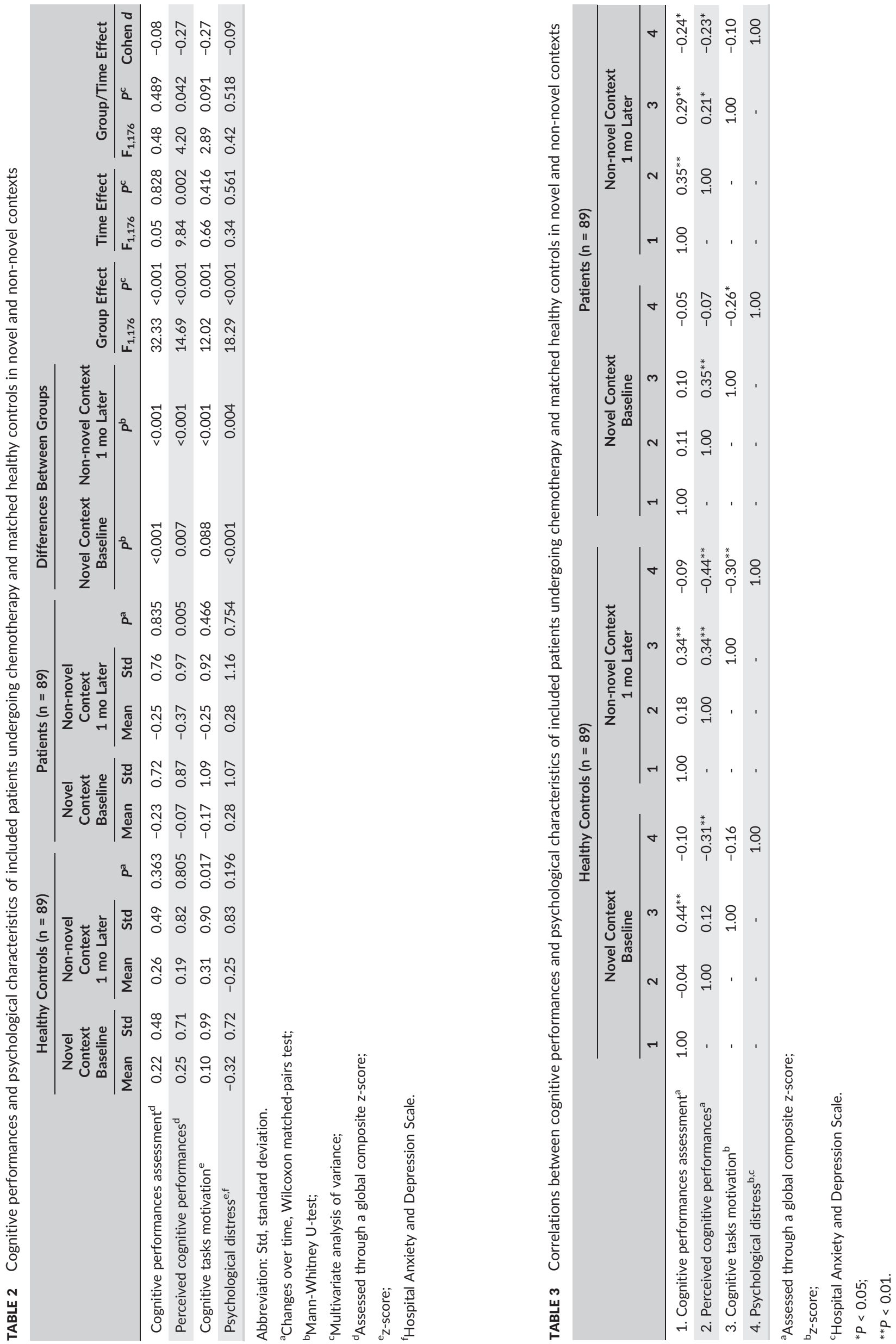


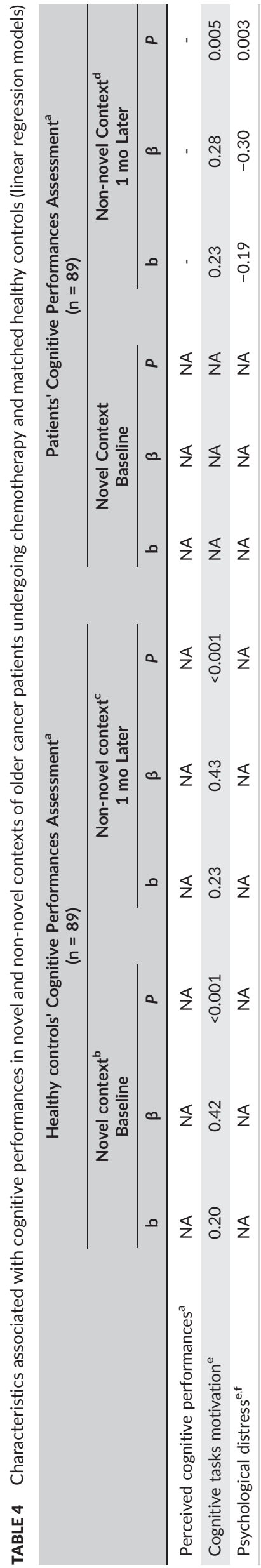

\subsection{Clinical implications}

Our results may have an important clinical implication: Healthcare professionals should be aware that clinically fit, older patients with hematologic malignancies undergoing chemotherapy already experience altered cognitive compensatory processes. To date, no recommendation has been made to support the cognitive compensatory processes of such patients. Based on the results of the current study, 2 recommendations could be made to optimize support. First, healthcare professionals should inform patients that, during treatment, their cognitive compensatory processes may be less effective, in order to normalize their subjective feelings. Second, patients should be informed that, although in a novel context it may be difficult for them to compensate for their altered cognitive functioning, increasing their motivation and regulating their psychological distress may improve their cognitive compensatory processes in a non-novel context. It should be recalled at this level the negative impact of distress on cognitive performance. ${ }^{29}$

\section{2 | Study limitations}

Some limitations of the study should be mentioned. First, as our study included only clinically fit patients with hematologic malignancies, our results may not be generalizable to more vulnerable populations or to patients with solid tumors. Second, patients were assessed only during the first month of chemotherapy treatment. Thus, our results do not reflect patients' later cognitive functioning. Third, by focusing on psychological factors, analyses performed did not control for other potential confounding factors, such as fatigue and pain.

\section{5 | CONCLUSION}

In conclusion, the results of this study indicate that older, clinically fit patients with hematological malignancies undergoing chemotherapy are at risk for altered cognitive compensatory processes in novel and non-novel contexts, suggesting that these processes should be addressed in cancer care. Although these results improve understanding of patients' cognitive compensatory processes, future studies should identify underlying mechanisms of such processes, to optimize potential revalidation interventions. Future studies should investigate the capacity of other physical, psychological, and social factors, such as physical exercise, perceived cognitive performance, and social support, to influence cognitive compensatory processes. Studies should also investigate the effects of altered cognitive compensatory processes on medical decision making, treatment compliance, daily functioning, and general QOL of patients. As to our knowledge, there are no other studies assessing cognitive compensatory processes in an older cancer patient population; the results of this study should of course be further studied. Finally, revalidation interventions that account for psychological factors, such as motivation and perceived cognitive performance, should be developed and tested to support the cognitive compensatory mechanisms of older patients with cancer.

\section{ACKNOWLEDGEMENTS}

This study was supported by the Fonds National de la Recherche Scientifique-Section Télévie of Belgium, the Université Libre de 
Bruxelles, the Université Catholique de Louvain, the Universite de Liège, and the Centre de Psycho-Oncologie. The authors thank the participating patients, investigators who conducted the assessments, and Mathieu Camelbeeck for their contribution.

\section{CONFLICT OF INTEREST}

The authors indicate no potential conflicts of interest.

\section{REFERENCES}

1. Butler KM, Mcdaniel MA, Dornburg CC, Price AL, Roediger HL. Age differences in veridical and false recall are not inevitable: the role of frontal lobe function. Psychon Bull Rev. 2004;11(5):921-925.

2. Wefel JS, Lenzi R, Theriault RL, Davis RN, Meyers CA. The cognitive sequelae of standard-dose adjuvant chemotherapy in women with breast carcinoma: results of a prospective, randomized, longitudinal trial. Cancer. 2004;100(11):2292-2299.

3. van Dam FS, Boogerd W, Schagen SB et al. Impairment of cognitive function in women receiving adjuvant treatment for high-risk breast cancer: high-dose versus standard-dose chemotherapy. J Natl Cancer Inst. 1998;90(3):210-218.

4. Maccormick RE. Possible acceleration of aging by adjuvant chemotherapy: a cause of early onset frailty? Med Hypotheses. 2006;67(2):212-215

5. Ahles TA, Saykin AJ. Candidate mechanisms for chemotherapy-induced cognitive changes. Nat Rev Cancer. 2007;7(3):192-201.

6. Jenkins V, Shilling V, Fallowfield L, Howell A, Hutton S. Does hormone therapy for the treatment of breast cancer have a detrimental effect on memory and cognition? A pilot study. Psychooncology. 2004;13(1):61-66.

7. Koppelmans V, Breteler MM, Boogerd W, Seynaeve C, Gundy C, Schagen SB. Neuropsychological performance in survivors of breast cancer more than 20 years after adjuvant chemotherapy. J Clin Oncol. 2012;30(10):1080-1086.

8. Kvale EA, Clay OJ, Ross-Meadows LA et al. Cognitive speed of processing and functional declines in older cancer survivors: an analysis of data from the ACTIVE trial. Eur J Cancer Care. 2010;19(1):110-117.

9. Yamada TH, Denburg NL, Beglinger LJ, Schultz SK. Neuropsychological outcomes of older breast cancer survivors: cognitive features ten or more years after chemotherapy. J Neuropsychiatry Clin Neurosci. 2010;22(1):48-54

10. Mohile SG, Lacy M, Rodin M et al. Cognitive effects of androgen deprivation therapy in an older cohort of men with prostate cancer. Crit Rev Oncol Hematol. 2010;75(2):152-159.

11. Hurria A, Goldfarb S, Rosen C et al. Effect of adjuvant breast cancer chemotherapy on cognitive function from the older patient's perspective. Breast Cancer Res Treat. 2006;98(3):343-348.

12. Extermann M. A comprehensive geriatric intervention detects multiple problems in older breast cancer patients. Crit Rev Oncol Hematol. 2004;49(1):69-75.

13. Chen H, Cantor A, Meyer J et al. Can older cancer patients tolerate chemotherapy? A prospective pilot study. Cancer. 2003;97(4):1107-1114.

14. Buckwalter JG, Crooks VC, Petitti DB. Cognitive performance of older women who have survived cancer. Int J Neurosci. 2005;115(9):1307-1314.
15. Ferguson RJ, McDonald BC, Saykin AJ, Ahles TA. Brain structure and function differences in monozygotic twins: possible effects of breast cancer chemotherapy. J Clin Oncol. 2007;25(25):3866-3870.

16. Schrijnemaekers AM, de Jager CA, Hogervorst E, Budge MM. Cases with mild cognitive impairment and Alzheimer's disease fail to benefit from repeated exposure to episodic memory tests as compared with controls. J Clin Exp Neuropsychol. 2006;28(3):438-455.

17. Posner MI, Snyder CR. Attention and Cognitive Control. In: Balota D \& Marsh E, eds. Cognitive Psychology: Key Readings. New York and Hove: Psychology Press; 2004:205-223.

18. Arndt J, Das E, Schagen SB, Reid-Arndt SA, Cameron LD, Ahles TA. Broadening the cancer and cognition landscape: the role of selfregulatory challenges. Psychooncology. 2014;23(1):1-8.

19. Suchy $Y$, Kraybill ML, Franchow E. Practice effect and beyond: reaction to novelty as an independent predictor of cognitive decline among older adults. J Int Neuropsychol Soc. 2011;17(01):101-111.

20. Katz S, Ford AB, Moskowitz RW, Jackson BA, Jaffe MW. Studies of illness in the aged: the index of ADL: a standardized measure of biological and psychosocial function. JAMA. 1963;185(12):914-919.

21. Roehrig B, Hoeffken K, Pientka L, Wedding U. How many and which items of activities of daily living (ADL) and instrumental activities of daily living (IADL) are necessary for screening. Crit Rev Oncol Hematol. 2007;62(2):164-171.

22. Wechsler D. Wechsler Adult Intelligence Scale-Fourth Edition (WAIS-IV). NCS Pearson: San Antonio, TX; 2008.

23. Grober E, Buschke H. Genuine memory deficits in dementia. Dev Neuropsychol. 1987;3(1):13-36.

24. Reitan RM. Validity of the Trail Making Test as an indicator of organic brain damage. Percept Mot Skills. 1958;8(3):271-276.

25. Joly F, Lange M, Rigal $O$ et al. French version of the Functional Assessment of Cancer Therapy-Cognitive Function (FACT-Cog) version 3. Support Care Cancer. 2012;20(12):3297-3305.

26. Wagner LI, Sweet J, Butt Z, Lai JS, Cella D. Measuring patient selfreported cognitive function: development of the functional assessment of cancer therapy-cognitive function instrument. J Support Oncol. 2009;7(6):W32-W39.

27. Zigmond AS, Snaith RP. The hospital anxiety and depression scale. Acta Psychiatr Scand. 1983;67(6):361-370.

28. Williams AM, Zent CS, Janelsins MC. What is known and unknown about chemotherapy-related cognitive impairment in patients with haematological malignancies and areas of needed research. $\mathrm{Br} J$ Haematol. 2016;174(6):835-846.

29. Asher A, Myers JS. The effect of cancer treatment on cognitive function. Clin Adv Hematol Oncol. 2015;13(7):441-450.

How to cite this article: Libert $Y$, Borghgraef C, Beguin Y, et al. Cognitive compensatory processes of older, clinically fit patients with hematologic malignancies undergoing chemotherapy: A longitudinal cohort study. Psycho-Oncology. 2017;26:2086-2093. https://doi.org/10.1002/pon.4424 